\title{
Are electronic white canes better than traditional canes? A comparative study with blind and blindfolded participants
}

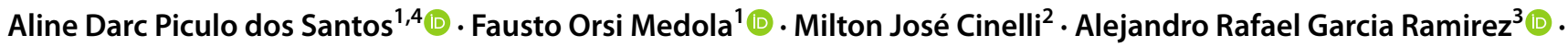 \\ Frode Eika Sandnes ${ }^{4,5}$ (i)
}

Published online: 17 February 2020

(c) The Author(s) 2020

\begin{abstract}
Visually impaired individuals often rely on assistive technologies such as white canes for independent navigation. Many electronic enhancements to the traditional white cane have been proposed. However, only a few of these proof-of-concept technologies have been tested with authentic users, as most studies rely on blindfolded non-visually impaired participants or no testing with participants at all. Experiments involving blind users are usually not contrasted with the traditional white cane. This study set out to compare an ultrasound-based electronic cane with a traditional white cane. Moreover, we also compared the performance of a group of visually impaired participants $(N=10)$ with a group of blindfolded participants without visual impairments $(N=31)$. The results show that walking speed with the electronic cane is significantly slower compared to the traditional white cane. Moreover, the results show that the performance of the participants without visual impairments is significantly slower than for the visually impaired participants. No significant differences in obstacle detection rates were observed across participant groups and device types for obstacles on the ground, while $79 \%$ of the hanging obstacles were detected by the electronic cane. The results of this study thus suggest that electronic canes present only one advantage over the traditional cane, namely in its ability to detect hanging obstacles, at least without prolonged practice. Next, blindfolded participants are insufficient substitutes for blind participants who are expert cane users. The implication of this study is that research into digital white cane enhancements should include blind participants. These participants should be followed over time in longitudinal experiments to document if practice will lead to improvements that surpass the performance achieved with traditional canes.
\end{abstract}

Keywords Electronic cane $\cdot$ Mobility $\cdot$ Blind and visually impaired $\cdot$ Assistive technology $\cdot$ Experimental procedures

\section{Introduction}

According to the World Health Organization [1], 285 million people are estimated to be visually impaired worldwide. According to data from the last census in Brazil [2], 18.8\% of the population stated having reduced vision. The most

Frode Eika Sandnes

frodes@oslomet.no

Aline Darc Piculo dos Santos

alinedarcps@gmail.com

Fausto Orsi Medola

fausto.medola@unesp.br

Milton José Cinelli

milton.cinelli@udesc.br

Alejandro Rafael Garcia Ramirez ramirez@univali.br common cases include cataracts, glaucoma, uncorrected refractive errors, diabetes, retinal displacement, ocular trauma, and aging-related diseases [1, 3, 4].

Visually impaired individuals experience many challenges in their daily activities, such as reading, practicing sports, socialization, getting an education and, especially,

1 Department of Design, Sao Paulo State University, Bauru, Brazil

2 Department of Design, Santa Catarina State University, Florianópolis, Brazil

3 Department of Computer Engineering, Univali, Itajaí, Brazil

4 Department of Computer Science, Oslo Metropolitan University, P.O. Box 4, St. Olavs plass, 0130 Oslo, Norway

5 Institute of Technology, Kristiania University College, Oslo, Norway 
moving around. The lack of vision has been found to have a direct impact on the gait pattern as well as on the walking speed [5-8]. Navigating independently, that is, without the help of others, is a major challenge for visually impaired individuals, which leads to frustration, low confidence, reduced autonomy, and physical safety risks [9]. To improve mobility, the visually impaired individual needs assistive technology (AT). According to Bhowmick and Hazarika [10], AT for visually impaired people is a research field that is gaining increasing prominence and has a very relevant social impact due to the increasing aging and blind populations.

Probably, the most common and accessible AT device for individuals without vision is the white cane. A white cane provides the users with tactile information about the surroundings thereby allowing these surroundings, especially on the ground, to be explored and obstacles to be detected, such as furniture, steps, etc. The white cane is also a symbolic device notifying onlookers that a person has reduced vision. This symbolic function is particularly useful in busy and crowded environments such as airports where people may be more careful toward the cane users. The symbolic function is also important when waiting for the bus altering the driver to stop and be extra vigilant during boarding.

Although the white cane helps the orientation and mobility for its users, it has a limited range and does not detect aerial obstacles such as tree branches and suspended trash cans [11-13]. This inevitably causes accidents, putting the users in physical danger [13]. According to a study by Manduchi and Kurniawan [14] of 300 blind participants, $40 \%$ reported head accidents at least once a year. Besides, they also reported that $23 \%$ of the incidents had medical consequences.

An Electronic Travel Aid (ETA) is a device that collects environmental information and transmits it to the user to allow independent movement [15]. Generally, ETAs detect obstacles in the user's path using one or more sensors and provide the information to the user through sounds, vibrations, or both $[16,17]$. The main advantage of an ETA over a regular white cane is its ability to identify obstacles above the waistline, therefore allowing more independent and safe mobility. While ETAs seem to benefit independent and safe mobility of visually impaired individuals, there is a lack of empirical evidence on the extent to which such devices can detect obstacles and improve the performance in mobility tasks.

There are few models of ETAs commercially available such as UltraCane (Sound Foresight Technology Ltd) and MiniGuide (American Printing House for the Blind, Inc.), both equipped with ultrasonic sensors [18, 19]. The main difference between them and the electronic cane used in this study relates to ergonomic concepts and costs [20]. The UltraCane and the MiniGuide devices cost approximately 2.4 and 1.5 times the estimated price of the electronic cane, respectively [18-20]. Unlike the MiniGuide device, the proposed electronic cane maintains the traditional cane handle, making it more convenient, since the MiniGuide cannot be used by itself, requiring the use along with a guide (human or dog), or cane, because it does not provide sufficient information to ensure safety [19]. Regarding the UltraCane, the proposed device only uses one ultrasonic sensor, thus having its operation simplified and its size, weight, and cost reduced [20].

Our review of the literature (see Sect. 2) revealed that many studies are carried out without visually impaired participants. One may question the validity of experiments involving non-visually impaired participants as practical substitutes for blind participants. Blind participants are experienced in interpreting the world using other senses and using white canes, while non-visually impaired individuals usually have little or no experience in being blindfolded. One of the objectives of this study was, therefore, to collect empirical evidence on any potential difference in performance between blind and blindfolded non-visually impaired users with the prediction that blind individuals would perform better due to their practice of being blind. Our first hypothesis was, therefore, the following:

Hypothesis 1 Blindfolding of non-visually impaired participants is an inaccurate substitute for blind participants in white cane experiments.

Our review of the literature also showed that the few studies that explored electronic white canes with blind participants did so without reference to the traditional white canes. Consequently, the research literature contains little empirical evidence to support the claim that electronic white canes pose improvements to the traditional canes. The second objective of this study was thus to assess if an electronic white cane that provides additional cues about obstacles poses an improvement over the white cane. We predicted that the electronic cane would lead to improved performance over the white cane. Our second hypothesis is thus the following:

Hypothesis 2 Electronic canes with additional obstacle cues lead to improved mobility performance over traditional passive white canes.

The novel contribution of this paper is thus captured in these two hypotheses that address issues that have not been sufficiently covered by the literature.

This paper is organized as follows: an overview of previous studies with ETAs is presented in Sect. 2. Section 3 describes the experimental setup, the materials and sample used, the tasks performed and how the data was 
analyzed. Section 4 presents the walking speed and obstacle detection results. The results are discussed in Sect. 5, and Sect. 6 concludes the paper with suggestions for future work.

\section{Related work}

Many technologies have been proposed through proofof-concept prototypes (see, for instance, Dakopoulos and Bourbakis's [21] survey on general assistive devices for the visually impaired, Tapu et al.'s [22] survey of wearable assistive devices for the visually impaired and Motta et al.'s [23] survey of electrical white canes). Unfortunately, many of the reported studies are technical system descriptions and do not include testing with actual users. These studies, therefore, provide little new insight into the operational suitability of these technologies. Examples of electronic white cane studies without evaluations with users include EyeCane [24, 25] which in essence is a distance measurement tool, Nada et al.'s [26] inexpensive devices based on an infrared sensor, Sheth et al.'s [27] ultrasound cane using prerecorded audio for feedback, Bouhamed et al.'s [28] cane using both ultrasound and a monochrome camera, Anwar and Aljahdali's [29] cane using five sensors including ultrasound, infrared, water detector, head and light detectors, Faria et al.'s [30] white cane with an RFID reader relying on RFID tags in the environment, Pallejà et al.'s [31] whisker-inspired white cane sensors, navigation with laser range scanners [32], flashlight-like scene exploration with a high frequency distance measurement tool [33], etc. Other researchers have addressed the feedback to the users more specifically, yet without documented user tests, for instance feedback via speech $[34,35]$, vibrations on different fingers to indicate navigation direction [36], advanced scene analysis with Google Tango [37], and the use of tractors on the arms [38].

Some studies proposing electronic white cane technologies have also conducted an experiment with blindfolded non-visually impaired users. Examples include Perera et al.'s [39] use of an ultrasound-based body area network with haptic feedback and text to speech. Unfortunately, the reported experiments are sketchy. Wang and Kuchenbecker [40] evaluated their HALO system on low hanging obstacles with 12 blindfolded participants, while Pyun et al. [41] employed 5 blindfolded participants. O'Brien et al. [42] evaluated a low-cost device that could be attached to an existing white cane with 16 blindfolded participants. Of these, 10 participants successfully detected more obstacles with the electric cane than the white cane, while 12 participants yielded slower walking speeds with the electronic cane. Another approach involves using colored tape on the floor which serves as guidelines with optical color detecting sensors on the cane in addition to RFID for absolution positioning. Shiizu et al. [43] evaluated such a system with 10 blindfolded participants, while Fukasawa and Magatani [44] evaluated a similar system with three blindfolded participants. Vera et al. [45] proposed to implement low-cost distance measurement with a laser pointer and a smartphone camera. The direction of the camera and the laser pointer are positioned at a non-parallel angle such that the laser pointer interaction with the scene is captured by the camera and the distance can be calculated. They evaluated their prototype using 16 blindfolded participants. A totally different approach was taken by Wachaja et al. [46] who implemented a smart walker for visually impaired users with reduced motor function based on recent advancements in robotics. Their system was tested with 9 blindfolded and one blind participant. Okazaki and Kajimoto [47] conducted an experiment with 11 blindfolded participants to explore the perception of distance through vibration.

Some studies also report observations of blind users using the technology. Hersh and Garcia Ramirez [48] conducted a two-part study with an ultrasound enhanced white cane. The first part of their experiment involved a prolonged use of the cane in a real urban environment at two locations in Brazil with five experienced cane users of which three were blind and two with low vision. They observed general satisfaction and usability. The second part involved a questionnaire sent to 18 cane users around Brazil of which five had low vision and 13 were blind. The results showed that half of the respondents liked the electronic enhancement of detecting objects above ground as it gave an increased sense of security to dangers. The respondents disliked that the devices could not be used in the rain and were difficult to fold. Suggested improvements included increased sensitivity and speed, to detect objects at leg height and to distinguish between different obstacle types. Menikdiwela, Dharmasena, and Abeykoon [49] evaluated a sonar-based device with vibrotactile feedback using three vibrators, with one vibrator for each sensor. Their experiment involved 7 blind schoolchildren that involved five parts addressing device interaction, learnability, walking between obstacles, obstacle height, and distance estimate. The results revealed an obstacle detection rate of $74 \%$, gaps down to $0.4 \mathrm{~m}$ could be detected, obstacles $0.1 \mathrm{~m}$ above ground could be detected and the distance estimate to vibration was $84 \%$. Their experiment was not well-structured, and it is hard to infer generalizations from the small number of participants. Williams et al. [50] studied the discrepancy between the feedback needed by blind individuals compared to nonvisually impaired individuals. Based on focus groups with 20 participants they found that blind individuals make use 
of small cues such as tiny bumps as they give indications of larger objects such as stairs. Sighted people prefer open places as they give a good overview, while such open spaces do not offer blind individuals enough cues such as walls and other objects. Blind individuals prefer "walking with others" by holding onto the arm of the sighted person and following their body motion. This is perceived as better than having to listen to instructions. Sighted people often make mistakes distinguishing left from right; they often provide ambiguous instructions such as "over there" and exhibit poor timing with instructions too early such as "stairs coming up" (at the other side of the room). Tzovaras et al. [51] employed 26 blind and visually impaired participants to assess a virtual reality system for training white cane skills to avoid training in dangerous environments such as heavy traffic. Zhao et al. [52] interviewed 13 low-vision individuals to identify how they navigate. They found that changes in the ground surface were the most important and that new technology should support depth judgments. Nicolau et al. [53] interviewed 8 blind individuals to gain insight into how they explore and interact with new places. Identifying faces and reading signs with small print during navigation has also been found to be among the most challenging tasks for lowvision individuals [54].

\section{Method}

\subsection{Experimental design}

Two experiments were conducted. The first experiment involved only non-visually impaired individuals in withingroups experimental design. The purpose of the first experiment was to obtain their preferred walking speed (PWS) under different conditions. Feedback mode was the independent variable with four levels, namely full visual feedback, blindfolded without a cane, blindfolded with the traditional white cane, and blindfolded with the electronic cane. Mean walking speed was the observed dependent variable.

The second experiment was performed using a mixed experimental design with two independent variables, namely device as a within-groups factor, and visual condition as a between-groups factor. The purpose was to contrast the two types of canes and two types of participants. The withingroups device factor had two levels, namely the traditional white cane and the electronic cane. The between-groups visual condition factor had two levels, visually impaired and blindfolded non-visually impaired participants. Walking speed and obstacle detection rates were the measured dependent variables. The order of the experiments with the canes was randomized to minimize learning effects among the blindfolded participants. The blind participants first used the white cane which they were already familiar with signaling their preferred walking speed, followed by the electronic cane.

\subsection{Participants}

We recruited ten visually impaired participants, who use the white cane for daily mobility, and 31 participants without visual impairments. All participants signed an informed consent form in which they were informed about the procedures and objectives of the research, as well as assured of the absence of any risks to their physical health and the possibility of leaving the experiment at any time.

The visually impaired participants (50\% female and 50\% male) had an average age of 46 years $(\mathrm{SD}=12.2)$ and an average height of $1.64 \mathrm{~m}(\mathrm{SD}=0.10)$. Of these, only one participant was left hand dominant. Regarding the physical activity level, 60\% stated having a low level and 40\% moderate level. Regarding ambulation, 10\% stated having dependent mobility, that is, using both cane and the company of a person to walk around, $80 \%$ stated to be independent, that is, moving around by themselves with only the cane and $10 \%$ declared to have both types of locomotion. In total, $90 \%$ of the participants used public transportation daily. All participants used the same type of white cane and tip.

Of the participants without visual impairments $48.4 \%$ were female and $51.6 \%$ male. The average age was 22.7 years $(\mathrm{SD}=3.67)$ and all were over 18 years old. The average height was $1.70 \mathrm{~m}(\mathrm{SD}=1.68)$. Of these, only $3.22 \%$ were left hand dominant. The participants were asked about their level of physical activity of which $48.4 \%$ stated having a low level, $48.4 \%$ moderate level and $3.2 \%$ intensive level. None of the participants had used a cane before.

\subsection{Materials}

Both a traditional white cane and an electronic white cane were used in the experiments both with the same tip (roller). The traditional white cane had a length of

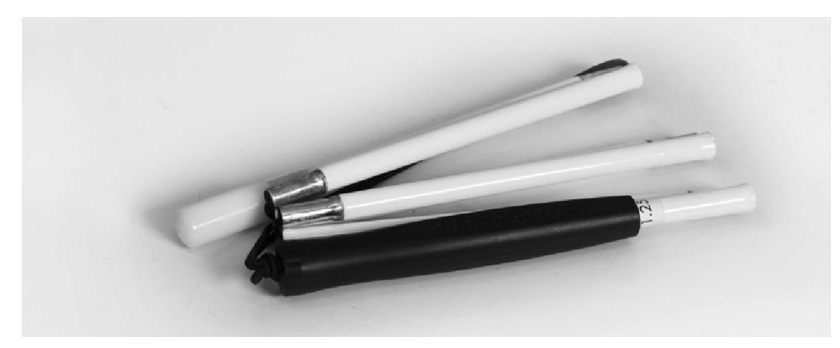

Fig. 1 Traditional white cane 


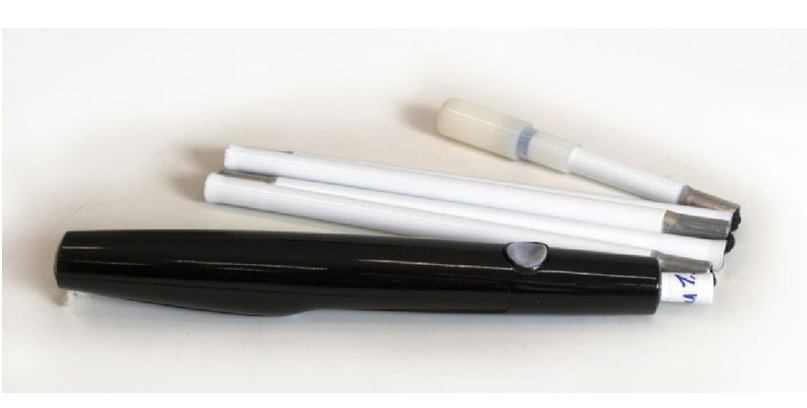

Fig. 2 Electronic cane

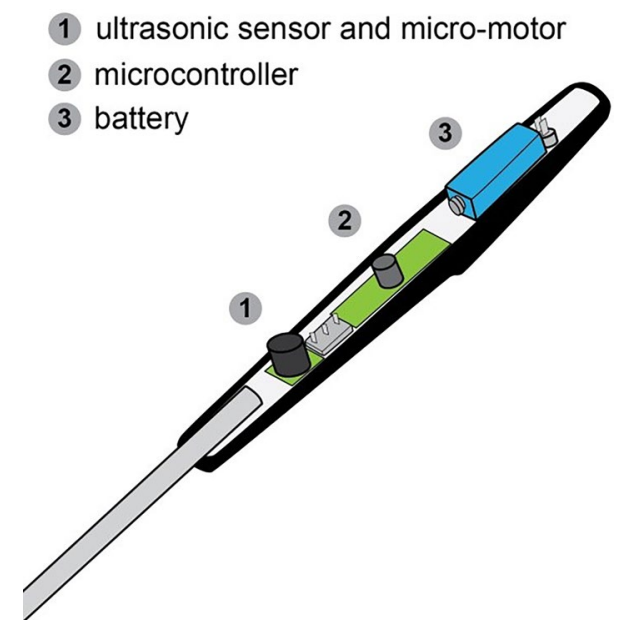

Fig. 3 Electronic cane components. Based on an illustration by Ramirez et al. [20]

$0.120 \mathrm{~m}$ and a mass of $0.255 \mathrm{~kg}$ excluding the tip. With the tip, the total length was $0.128 \mathrm{~m}$ with a total mass of $0.297 \mathrm{~kg}$ (Fig. 1). This cane has three main parts: a handle (grip) made from anatomic rubber, a straight aluminum shaft, and a nylon tip that is in direct contact with the object or the ground. The electronic cane had a length of $0.1255 \mathrm{~m}$ with a mass of $0.305 \mathrm{~kg}$ without the tip, and a total length of $0.131 \mathrm{~m}$ and a total mass of $0.347 \mathrm{~kg}$ including the tip). The electronic cane was developed at UNIVALI in collaboration with UDESC (Fig. 2). It has a micro-motor for creating vibrotactile feedback, a $9 \mathrm{~V}$ battery and an integrated ultrasonic sensor that identifies obstacles above the waistline at a range of $1.5 \mathrm{~m}$ within a sector of $30^{\circ}$. The user is alerted through vibration or sound whenever an obstacle is detected (Fig. 3).

As the user approaches the obstacle with the electronic cane, the feedback increases accordingly [20]. According to Ramirez et al. [20], this device was created to avoid the limitation of the traditional white cane in detecting obstacles above the user's waistline and, therefore, to achieve better spatial exploration. The device uses ultrasonic echo to detect physical barriers and the echo detection can be performed in real time on small portable devices.

\subsection{Experimental procedure}

The walking test was developed based on an experimental setup reported by Jeong and Yu [15] and Roentgen et al. [55]. It comprised two parts. In part one, the walking performance of the individuals was measured without obstacles. The participants were first instructed to walk back and forth along the path without obstacles to get familiarized with the path in their self-selected walking speed. The participants were instructed on how to use both devices and were allowed 5 min for familiarization. The instructions included: how to hold and position the cane (in front of the body), how to use the cane (rolling from side to side), how to turn on and off the electronic cane, and how the electronic cane emits feedback as approaches the obstacles (increasing the sound and vibration). Since the visually impaired participants are already used to the white cane, they only received training regarding the electronic cane. We observed that, although the orientation and mobility course teaches that the white cane should be positioned in front of the body, the visually impaired participants used it on the side of the body. The first experiment with the non-visually impaired participants was conducted under four conditions, namely with full vision and no cane, blindfolded without a cane, blindfolded with the traditional cane and blindfolded with the electronic cane. Swimming glasses covered in black tape were used to blindfold the participants.

Experiment two involved a walking test with seven obstacles. There were two types of obstacles: two obstacles suspended from the ceiling and five on the ground. The participants were given an indoor training session involving two obstacles in different sizes and heights (Figs. 4, 5) in a space of $5 \times 2 \mathrm{~m}$. The actual experiment was conducted outdoors on a path with outer dimensions of $21 \times 2.5$ (Fig. 6), with seven obstacles. Then the participants were asked to walk the path with obstacles along one direction only. They were positioned in the center of the path and instructed to wait for the research to inform they could start. They were also instructed not to touch the obstacles, relying only on the cane feedback to detect them and to follow the tactile floor path with the cane. Also, if they detect any obstacle along the way, they should avoid it and go back to the path (Fig. 7). After completing the task with the first cane, they repeated the task with the second cane.

The positions of the obstacles were marked on the floor so that all the different trials would have the same conditions. 
Fig. 4 Height, width, depth, and vertical position of the obstacles
TRAINING ROOM

1) $118.0 \times 38.5 \times 28.0 \mathrm{~cm}$

2. $40.0 \times 32.0 \times 27.0 \mathrm{~cm}$

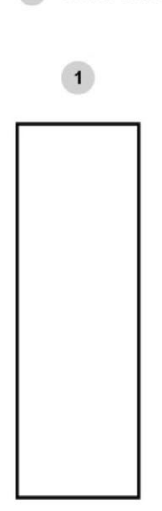

\section{2}
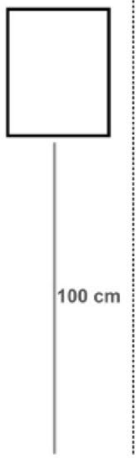

$0 \times 30.0 \times 40.0 \mathrm{~cm}$

(3) $97.5 \times 19.5 \times 26.5 \mathrm{~cm}$

(4) $41.0 \times 31.5 \times 27.0 \mathrm{~cm}$

(5) $133.0 \times 28.0 \times 23.5 \mathrm{~cm}$

(6) $79.0 \times 31.5 \times 26.0 \mathrm{~cm}$

$733.5 \times 63.0 \times 33.5 \mathrm{~cm}$

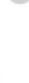

1

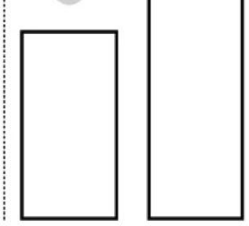

\section{EXPERIMENT PATH}

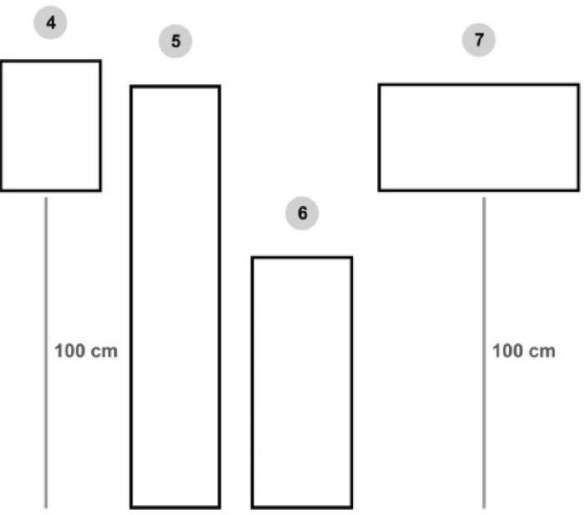

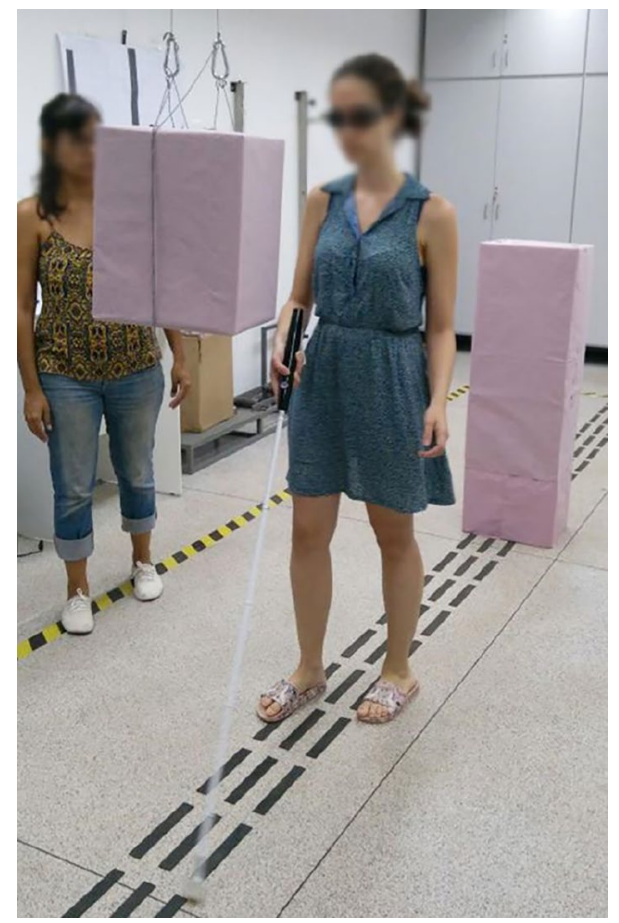

Cardboard obstacles were made to represent real obstacles that can be found in a walking environment such as tree branches and suspended trashcans that are not identified by the traditional canes. Both the training room and the outdoor path contained tactile floor paths to help the participants orientate along a straight line.

The hanging obstacles were not included for the visually impaired participants in the white cane condition (second part of the experiment) as it would not be possible to detect these obstacles with the white cane. Walking into these obstacles could have caused unpleasant embarrassment among the visually impaired participants.

\subsection{Data analysis}

The mean walking speed in meters per second was computed by dividing the path length $(21 \mathrm{~m})$ by the measured total time to complete the path. The percentage of obstacles detected by the white canes was acquired by manually inspecting video recordings of the participants. Statistical analyses were performed using the JASP version 0.9.1.0 [56].

Fig. 5 Experimental setup in the training room

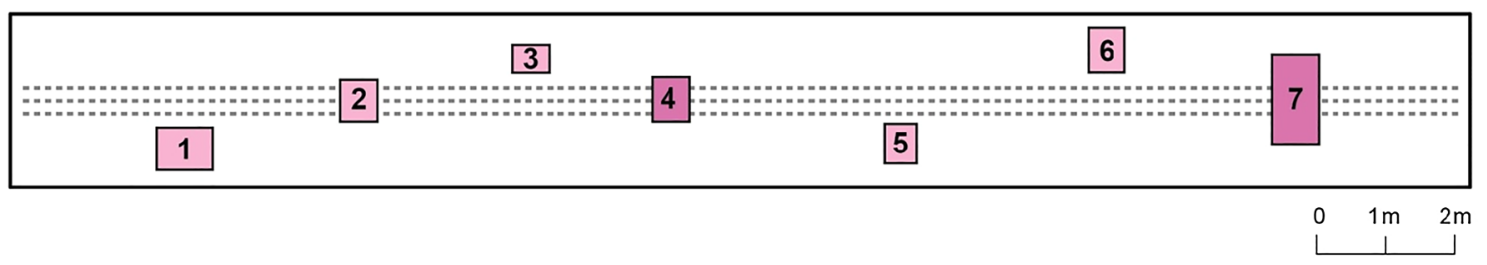

Fig. 6 Path with the positions of the outdoor obstacles 


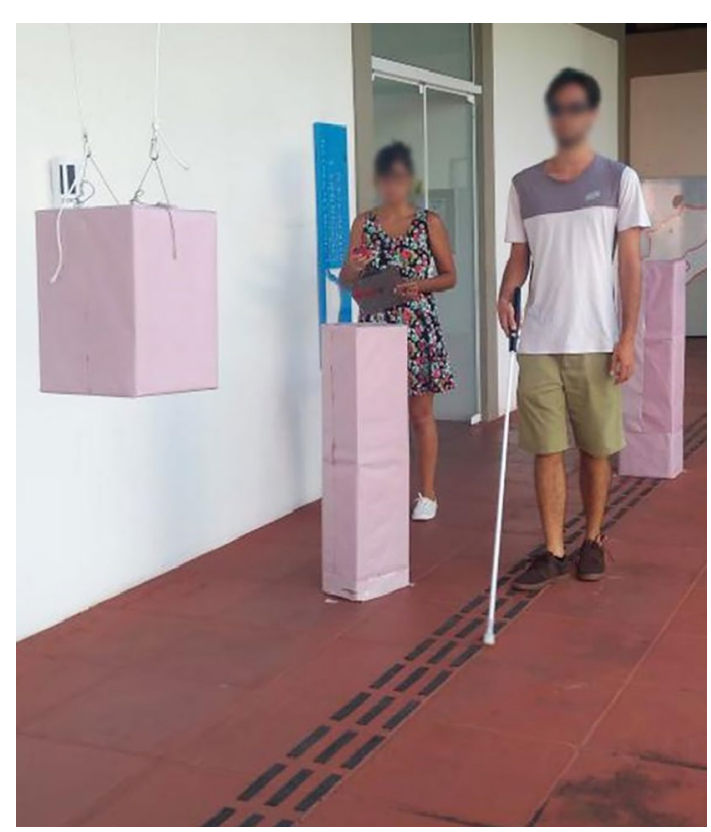

Fig. 7 Data collection. Experiment path

\subsection{Ethics}

This study was approved (Process N. 2.258.364) by the Ethics in Research Committee of Faculty of Architecture, Arts and Communication, of São Paulo State University (UNESP) - Bauru, Brazil, attending the Resolution N. 510/2016 of the National Council of Health. All participants signed an informed consent form in which they were informed about the procedures and objectives of the research, as well as assured of the absence of any risks to their physical health and the possibility of leaving the experiment without any prejudice. Video recordings were deleted after the data analysis. This research was

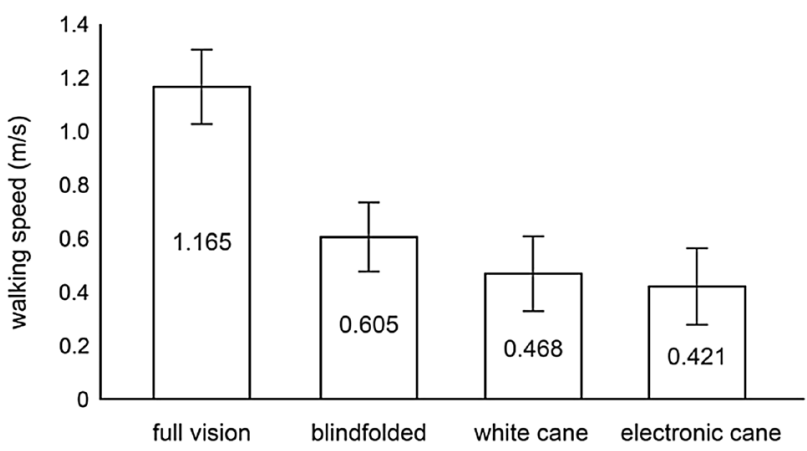

Fig. 8 Walking speed observed in the four feedback conditions without obstacles conducted before the introduction of the General Data Protection Regulation (GDPR).

\section{Results}

\subsection{Walking speed}

Figure 8 shows the results from the first part of the experiment. The measurements were checked for normality. Mauchly's test revealed that the data did not satisfy the assumption of sphericity ( $W=0.479, p<.001$ ). Greenhouse-Geisser and the Huynh-Feldt epsilon $(\varepsilon)$ values were both below 0.75 and Greenhouse-Geisser corrections were therefore applied to the repeated measures ANOVA. The repeated measures ANOVA revealed that there was a significant effect of condition $[F(2.049,61.468)=246.6$, $\left.p<.001, \eta^{2}=.892\right]$.

The walking speed in meters per second in normal conditions, that is, walking with full vision without a cane (the preferred walking speed PWS), was the highest $(M=1.17, \mathrm{SD}=0.14)$. The absence of the vision (blindfolded without a cane) reduced the walking speed by $51 \%$ of the PWS to $(M=0.60 \mathrm{~m} / \mathrm{s}, \mathrm{SD}=0.13)$. Bonferroni post hoc tests revealed that this difference was significant $(p<.001)$. The walking speeds with both types of canes were slower than without the cane, and these differences were also significantly different $(p<.001)$. The results obtained with the white cane were marginally faster $(M=0.47 \mathrm{~m} / \mathrm{s}, \mathrm{SD}=0.14)$ than the electronic cane $(M=0.42 \mathrm{~m} / \mathrm{s}, \mathrm{SD}=0.14)$, but these walking speeds were not significantly different $(p=.103)$.

The observations for the second part of the experiment were checked for both normality and equality of variances [Levene's tests gave $(F(1,39)=1.583, p=.216)$ for the white cane and $(F(1,39)=3.925, p=.055)$ for the elec-

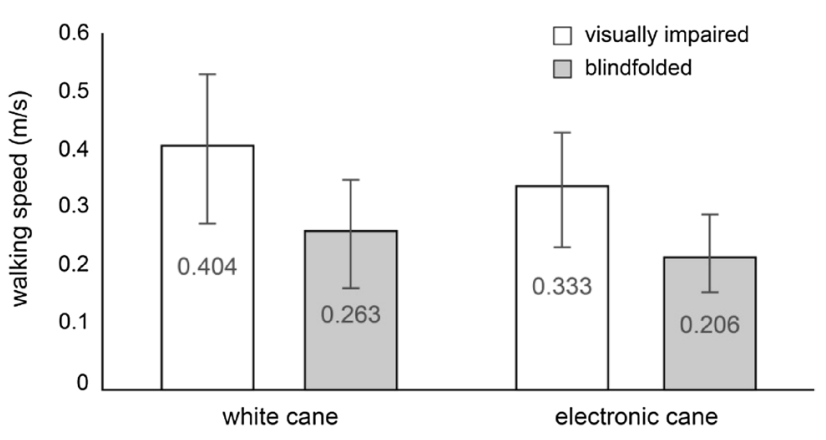

Fig. 9 Walking speed using the canes with obstacles

tronic cane]. A repeated measures ANOVA revealed that there were both a significant within-groups effect of cane 
$\left[F(1,39)=43.075, p<.001, \eta^{2}=.521\right]$ and also a significant between-groups effect of experience with canes $[F(1$, 39) $\left.=22.16, p<.001, \eta^{2}=.362\right]$.

Figure 9 shows the results. Clearly, the white cane yielded a faster walking speed (in meters per second) for both the visually impaired $(M=0.40, \mathrm{SD}=0.11)$ and the blindfolded participants $(M=0.26, \mathrm{SD}=0.08)$ than the electronic cane which resulted in a slower walking speed for both the visually impaired $(M=0.33, \mathrm{SD}=0.10)$ and the blindfolded participants $(M=0.21, \mathrm{SD}=0.07)$. Moreover, the visually impaired participants were faster than the blindfolded participants. No interactions were observed between cane type and experience with cane $[F(1,39)=0.59, p=.458]$.

\subsection{Obstacle detection}

The obstacle detection rate observations did not comply with the assumptions of the parametric statistical tests. The measurements were, therefore, transformed using the adjusted rank transform (ART) procedure [57]. The transformed data could then be analyzed using a parametric mixed factorial ANOVA. The test revealed that there was no significant effect of cane type $[F(1,39)=0.078, p=.781]$ and there was no significant effect of participant type $[F(1,39)=0.011$, $p=.917]$. Moreover, no interaction effect was observed across cane type and participant type $[F(1,39)=2.056$, $p=.159]$.

The white cane detected on average $67.8 \%$ of the ground obstacles. The blind users detected more obstacles $(M=72.0 \%, \mathrm{SD}=19.0 \%)$ than the blindfolded participants $(M=66.5 \%, \mathrm{SD}=23.9 \%)$, although this difference was not significant.

The electronic cane detected only $64.9 \%$ of the ground obstacles. The visually impaired users actually detected fewer obstacles $(M=60.0 \%, \mathrm{SD}=24.9 \%)$ than the blindfolded participants $(M=66.5 \%, \mathrm{SD}=20.0 \%)$. Again, the type of participant was not a significant factor.

A detailed analysis of the results obtained with the electronic cane showed that only $26.7 \%$ of the ground obstacles were detected by the ultrasonic sensor. Again, the blindfolded participants detected more obstacles with the ultrasonic sensor $(M=29.1 \%, \mathrm{SD}=19.5 \%)$ than the visually impaired participants $(M=19.5 \%, \mathrm{SD}=22.9 \%)$. A Mann-Whitney $U$ test showed that this difference was not significant $(W=115.0, p=.216)$.

On average, $75.6 \%$ of the hanging obstacles were successfully detected with the electronic cane of which none could be detected by the white cane. Of these, the blindfolded participants detected more obstacles with the ultrasonic sensor $(M=79.0 \%, \mathrm{SD}=28.2 \%)$ than the visually impaired participants $(M=65.0 \%, \mathrm{SD}=41.1 \%)$. A Mann-Whitney $U$ test showed that this difference was not significantly different $(W=128.0, p=.356)$.

\section{Discussion}

Mobility is important for activity and social participation, and evidence indicating that participation is affected in visually impaired persons has been reported [58]. Therefore, investigating factors that influence mobility in this population is important as it supports addressing social participation. In this context, the present study provides additional knowledge on the effect of two assistive devices - white cane and ultrasound-based electronic cane-on the mobility performance.

As expected, the absence of vision reduced the walking speed among the non-visually impaired participants. This may be explained by the importance of the visual system for mobility. Vision provides vital information about the environment while moving around allowing stability and path planning [5]. The results agree with those of Hallemans et al. [5] in that situations where vision is compromised result in different adaptations to the gait pattern such as the reduction of the walking speed, shorter steps, and deviation of straight paths.

The observed walking speed was slower with canes compared to not using canes among the blindfolded individuals. This can be explained by the inexperience of the participants using white canes. It must be taken into consideration that the participants in this study had uncorrected or close to uncorrected vision. Comparatively, the human physiological senses of hearing and touch among blind individuals are more trained. Blindfolding individuals with uncorrected vision is not a fully realistic substitute for blind participants. Sounds, vibrations, and noises that are easily perceived by blind individuals may have not been noticed by the participants, making these participants more careful and slower. The adaptation for the lack of vision demands attention to other senses, and this learning process takes time to integrate the sensorial information in a way to reach optimal mobility performance.

The visually impaired participants exhibited a faster walking speed compared to the blindfolded participants. One may speculate whether the presence of the hanging obstacles for the blindfolded participants in the white cane conditions slowed these participants down. However, there were only two hanging obstacles and the same pattern was also present in the electronic cane condition which comprised identical obstacle configurations. It is, therefore, likely that the faster walking speed among the visually impaired participants is mainly attributed to their practice and established skills using white canes. 
The observed walking speed with the white cane was faster than the electronic cane for both participant groups. One possible explanation could be the simplicity of the regular cane that relies on haptic feedback the participants were used to. Since the electronic cane emits unfamiliar sound and vibrotactile feedback, the participants had to learn to interpret the signals, and this might have demanded more attention, influencing the participants' walking speed. The cognitive load has been indicated as one of the factors that contribute to fatigue in adults with visual impairments [59]. Therefore, simple and easy-tolearn assistive devices are recommended.

Throughout the experiments, it was possible to observe some limitations among the non-visually impaired participants such as the lack of experience with having no visual information as well as with the use of both canes, since none of these participants had used a cane before.

Therefore, the results for walking speed, which also agree with observations made by O'Brien et al. [42], seem to confirm hypothesis 1 , namely that blindfolding non-visually impaired participants is an inaccurate substitute for blind participants in white cane experiments. Results obtained with non-visually impaired users will not necessarily generalize to visually impaired users. Visually impaired and non-visually impaired users have developed different skills which, for instance, are exhibited in different gait patterns (see Hallemans et al. [5]). The skills of visually impaired users cannot be simulated simply through blindfolding nonvisually impaired users. Consequently, blindfolding may not be advisable in such experiments. One exception may arise if one needs to contrast users with different skill sets.

In terms of obstacle detection, the results confirmed that the electronic cane can detect most of the hanging obstacles which cannot be detected by the white cane. However, in terms of ground obstacles, no significant differences could be found between the two types of canes or across the participant groups, although the blindfolded participants performed better than the blind participants. One may speculate that users who are not used to the white cane are more quickly able adapted to the electronic cane as they are not used to the white cane. If this is the case, one may speculate that electronic canes may be a more suitable tool for individuals losing their vision with age. However, such a conclusion cannot be drawn on the current data. The lack of significant results may be due to the low number of obstacles of each type. In order to get more reliable results, more obstacles should be included in the experiments.

Based on these results, which also agree with observations by O'Brien et al. [42], we can only partially accept hypothesis 2 , namely that electronic canes with additional obstacle cues lead to improved performance over traditional passive white canes, as the only observed benefit were the ability to detect hanging obstacles which cannot be detected by the white canes. In terms of ground obstacles, hypothesis 2 is rejected.

We observed that the time provided to learn the functionality of the electronic cane might have been insufficient for the participants to fully understand the system. Another limitation was the shortness of the path that on average took less than a minute to complete. The task took approximately 2 min with obstacles. A study with more trials and/or more time of use can bring more information about the adaption and learning process. In terms of research design, the results suggest that there were too few obstacles of both types to get reliable measurements. More obstacles may also be needed as this type of observation demands nonparametric tests which have less power than parametric tests. Future work should ensure enough training for the participants both with lack of vision as well as the use of the cane. Also, a longer and more challenging path with more obstacles of both types is recommended.

\section{Conclusions}

This study compared the white cane to an ultrasoundbased electronic cane on both visually impaired and nonvisually impaired blindfolded participants. The results show that walking speed with the electronic cane is significantly slower compared to the traditional white cane. Moreover, the results show that the performance of the participants without visual impairments is significantly slower than for the visually impaired participants. No significant differences in obstacle detection rates were observed across participant groups and device types for obstacles on the ground. A total of $79 \%$ of the hanging obstacles that cannot be detected by the white cane were detected by the electronic cane. These results suggest that electronic canes present only one advantage over the traditional cane, namely in its ability to detect hanging obstacles. Therefore, this study shows that the technology used in the electronic cane, namely the ultrasound sensor, has the potential to help the visually impaired people that need an assistive technology on a daily basis by allowing a safer navigation, as it should prevent collisions with obstacles above the waistline which are not detected by the traditional white cane. Moreover, the results show that blindfolded participants are insufficient substitutes for blind participants in such an experiment. Longitudinal experiments should also be conducted to document how practice leads to improvements. The results also showed nonsignificant differences across the participant groups as the non-visually impaired seem to be better at exploiting the technologically enhanced electronic cane. Future work should, therefore, explore the group differences with 
a more elaborate experimental setup to assess if blind individuals are negatively affected by their white cane skills. If this is the case, electronic canes may be especially useful for individuals who lose their vision later in life.

Acknowledgements Open Access funding provided by OsloMet - Oslo Metropolitan University. This research was funded by CAPES. The authors would also like to thank the Norwegian Centre for International Cooperation in Education (UTF-2016-long-term/10053) for financial support.

\section{Compliance with ethical standards}

Conflict of interest On behalf of all authors, the corresponding author states that there is no conflict of interest.

Open Access This article is licensed under a Creative Commons Attribution 4.0 International License, which permits use, sharing, adaptation, distribution and reproduction in any medium or format, as long as you give appropriate credit to the original author(s) and the source, provide a link to the Creative Commons licence, and indicate if changes were made. The images or other third party material in this article are included in the article's Creative Commons licence, unless indicated otherwise in a credit line to the material. If material is not included in the article's Creative Commons licence and your intended use is not permitted by statutory regulation or exceeds the permitted use, you will need to obtain permission directly from the copyright holder. To view a copy of this licence, visit http://creativecommons.org/licenses/by/4.0/.

\section{References}

1. World Health Organization: Visual impairment and blindness. World Health Organization. Fact Sheet n. 282 (2014). http://www. who.int/mediacentre/factsheets/fs282/en/. Accessed 13 Jan 2016

2. IBGE - Instituto Brasileiro De Geografia E Estatística. Censo Demográfico 2010. Características Gerais da População, Religião e Pessoas com Deficiência: Publicação Completa. IBGE, Rio de Janeiro (2011)

3. Gil, N.: Deficiência Visual. MEC. Secretaria de Educação a Distância, Brasília (2000)

4. Adebiyi, A., Sorrentino, P., Bohlool, S., Zhang, C., Arditti, M., Goodrich, G., Weiland, J.D.: Assessment of feedback modalities for wearable visual aids in blind mobility. PLoS ONE 12(2), e0170531 (2017)

5. Hallemans, A., Ortibus, E., Meire, F., Aerts, P.: Low vision affects dynamic stability of gait. Gait Posture 32(4), 547-551 (2010)

6. Reynard, F., Terrier, P.: Role of visual input in the control of dynamic balance: variability and instability of gait in treadmill walking while blindfolded. Exp. Brain Res. 233(4), 1031-1040 (2015)

7. Cuturi, L.F., Aggius-Vella, E., Campus, C., Parmiggiani, A., Gori, M.: From science to technology: orientation and mobility in blind children and adults. Neurosci. Biobehav. Rev. 71, 240-251 (2016)

8. Wong, S.: Traveling with blindness: a qualitative space-time approach to understanding visual impairment and urban mobility. Health Place 49, 85-92 (2018)

9. Jafri, R., Khan, M.M.: User-centered design of a depth data based obstacle detection and avoidance system for the visually impaired. Human-centric Comput. Inf. Sci. 8(1), 14 (2018)

10. Bhowmick, A., Hazarika, S.M.: An insight into assistive technology for the visually impaired and blind people: state-of-the-art and future trends. J. Multimodal User Interfaces 11(2), 149-172 (2017)

11. Lugli, D., Suemitsu, K., Minozzo, M., Okimoto, M.L.: Bengala customizável para mulheres com deficiência visual. Des. Tecnol. 6(12), 44-53 (2016)

12. Gao, Y., Chandrawanshi, R., Nau, A.C., Tse, Z.T.H.: Wearable virtual white cane network for navigating people with visual impairment. Proc. Inst. Mech. Eng. [H] 229(9), 681-688 (2015)

13. Kim, S.Y., Cho, K.: Usability and design guidelines of smart canes for users with visual impairments. Int. J. Des. 7(1), 99-110 (2013)

14. Manduchi, R., Kurniawan, S.: Mobility-related accidents experienced by people with visual impairment. AER J. 4(2), 44-54 (2011)

15. Jeong, G.Y., Yu, K.H.: Multi-section sensing and vibrotactile perception for walking guide of visually impaired person. Sensors 16(7), 1070 (2016)

16. Elmannai, W., Elleithy, K.: Sensor-based assistive devices for visually-impaired people: current status, challenges, and future directions. Sensors 17(3), 565 (2017)

17. Kiuru, T., Metso, M., Utriainen, M., Metsävainio, K., Jauhonen, H.M., Rajala, R., Savenius, R., Ström, M., Jylhä, T.N., Juntunen, R., Sylberg, J.: Assistive device for orientation and mobility of the visually impaired based on millimeter wave radar technology—clinical investigation results. Cogent Eng. 5(1), 1450322 (2018)

18. Sound Foresight Technology Ltd: UltraCane (2019). https://www. ultracane.com/ultracanecat. Accessed 28 Mar 2019

19. American Printing House for the Blind, Inc: Miniguide US (2019). https://shop.aph.org/webapp/wcs/stores/servlet/Product_Minig uide\%20US_1189771P_10001_11051. Accessed 28 Mar 2019

20. Ramirez, A.R.G., da Silva, R.F.L., Cinelli, M.J., de Albornoz, A.D.C.: Evaluation of electronic haptic device for blind and visually impaired people: a case study. J. Med. Biol. Eng. 32(6), 423-428 (2012)

21. Dakopoulos, D., Bourbakis, N.G.: Wearable obstacle avoidance electronic travel aids for blind: a survey. IEEE Trans. Syst. Man Cybern. Part C (Appl. Rev.) 40(1), 25-35 (2010)

22. Tapu, R., Mocanu, B., Zaharia, T.: Wearable assistive devices for visually impaired: a state of the art survey. Pattern Recognit. Lett. (2018). https://doi.org/10.1016/j.patrec.2018.10.031

23. Motta, G., Ma, T., Liu, K., Pissaloux, E., Yusro, M., Ramli, K., Connier, J., Vaslin, P., Li, J.J., De Vaulx, C., Shi, H.: Overview of smart white canes: connected smart cane from front end to back end. In: Pissaloux, E., Velazquez, R. (eds.) Mobility of Visually Impaired People, pp. 469-535. Springer, Cham (2018)

24. Ju, J.S., Ko, E., Kim, E.Y.: EYECane: navigating with camera embedded white cane for visually impaired person. In: Proceedings of the 11th International ACM SIGACCESS Conference on Computers and Accessibility. ACM, pp. 237-238 (2009)

25. Buchs, G., Maidenbaum, S., Amedi, A. Augmented non-visual distance sensing with the EyeCane. In: Proceedings of the 6th Augmented Human International Conference. ACM, pp. 209-210 (2015)

26. Nada, A.A., Fakhr, M.A., Seddik, A.F.: Assistive infrared sensor based smart stick for blind people. In: Science and Information Conference (SAI), 2015. IEEE, pp. 1149-1154 (2015)

27. Sheth, R., Rajandekar, S., Laddha, S., Chaudhari, R.: Smart white cane-an elegant and economic walking aid. Am. J. Eng. Res. 3(10), 84-89 (2014)

28. Bouhamed, S.A., Kallel, I.K., Masmoudi, D.S.: New electronic white cane for stair case detection and recognition using ultrasonic sensor. Int. J. Adv. Comput. Sci. Appl. 4(6), 243-255 (2013)

29. Anwar, A., Aljahdall, S.: A smart stick for assisting visually impaired people. J. Theor. Appl. Inf. Technol. 96(14), 86-90 (2018) 
30. Faria, J., Lopes, S., Fernandes, H., Martins, P., Barroso, J.: Electronic white cane for blind people navigation assistance. In: World Automation Congress (WAC), 2010. IEEE, pp. 1-7 (2010)

31. Pallejà, T., Tresanchez, M., Teixidó, M., Palacin, J.: Bioinspired electronic white cane implementation based on a LIDAR, a triaxial accelerometer and a tactile belt. Sensors 10(12), 1132211339 (2010)

32. Gomez, J.V., Sandnes, F.E.: RoboGuideDog: Guiding blind users through physical environments with laser range scanners. Procedia Comput. Sci. 14, 218-225 (2012)

33. Yuan, D., Manduchi, R.: Dynamic environment exploration using a virtual white cane. In: IEEE Computer Society Conference on Computer Vision and Pattern Recognition, 2005. CVPR 2005, vol. 1. IEEE, pp. 243-249 (2005)

34. Stent, A.J., Azenkot, S., Stern, B.: Iwalk: a lightweight navigation system for low-vision users. In: Proceedings of the 12th International ACM SIGACCESS Conference on Computers and Accessibility. ACM, pp. 269-270 (2010)

35. Lee, J.S., Choi, H., Lee, J.: TalkingCane: designing interactive white cane for visually impaired people's bus usage. In: Proceedings of the 17th International Conference on Human-Computer Interaction with Mobile Devices and Services Adjunct. ACM, pp. 668-673 (2015)

36. Khan, M.F., Kumar, A.: White cane navigation using Arduino Uno. In: Intelligent Communication, Control and Devices. Springer, Singapore, pp. 1719-1727 (2018)

37. Jafri, R., Campos, R.L., Ali, S.A., Arabnia, H.R.: Visual and infrared sensor data-based obstacle detection for the visually impaired using the Google Project Tango Tablet development kit and the unity engine. IEEE Access 6, 443-454 (2018)

38. Kammoun, S., Jouffrais, C., Guerreiro, T., Nicolau, H., Jorge, J.: Guiding blind people with haptic feedback. In: Frontiers in Accessibility for Pervasive Computing (Pervasive 2012), vol. 3 (2012)

39. Pereira, A., Nunes, N., Vieira, D., Costa, N., Fernandes, H., Barroso, J.: Blind Guide: an ultrasound sensor-based body area network for guiding blind people. Procedia Comput. Sci. 67, 403-408 (2015)

40. Wang, Y., Kuchenbecker, K.J.: HALO: haptic alerts for low-hanging obstacles in white cane navigation. In: 2012 IEEE Haptics Symposium (HAPTICS). IEEE, pp. 527-532 (2012)

41. Pyun, R., Kim, Y., Wespe, P., Gassert, R., Schneller, S.: Advanced augmented white cane with obstacle height and distance feedback. In: 2013 IEEE International Conference on Rehabilitation Robotics (ICORR). IEEE, pp. 1-6 (2013)

42. O'Brien, E.E., Mohtar, A.A., Diment, L.E., Reynolds, K.J.: A detachable electronic device for use with a long white cane to assist with mobility. Assist. Technol. 26(4), 219-226 (2014)

43. Shiizu, Y., Hirahara, Y., Yanashima, K., Magatani, K.: The development of a white cane which navigates the visually impaired. In: 29th Annual International Conference of the IEEE Engineering in Medicine and Biology Society, 2007. EMBS 2007. IEEE, pp. 5005-5008 (2007)

44. Fukasawa, A.J., Magatani, K.: A navigation system for the visually impaired an intelligent white cane. In: 2012 Annual International Conference of the IEEE Engineering in Medicine and Biology Society (EMBC). IEEE, pp. 4760-4763 (2012)

45. Vera, P., Zenteno, D., Salas, J.: A smartphone-based virtual white cane. Pattern Anal. Appl. 17(3), 623-632 (2014)
46. Wachaja, A., Agarwal, P., Zink, M., Adame, M.R., Möller, K., Burgard, W.: Navigating blind people with walking impairments using a smart walker. Auton. Robots 41(3), 555-573 (2017)

47. Okazaki, R., Kajimoto, H.: Perceived distance from hitting with a stick is altered by overlapping vibration to holding hand. In: CHI' 14 Extended Abstracts on Human Factors in Computing Systems. ACM, pp. 1903-1908 (2014)

48. Hersh, M.A., García Ramírez, A.R.: Evaluation of the electronic long cane: improving mobility in urban environments. Behav. Inf. Technol. 37(12), 1203-1223 (2018)

49. Menikdiwela, M.P., Dharmasena, K.M.I.S., Abeykoon, A.H.S.: Haptic based walking stick for visually impaired people. In: 2013 International conference on Circuits, Controls and Communications (CCUBE). IEEE, pp. 1-6 (2013)

50. Williams, M.A., Galbraith, C., Kane, S.K., Hurst, A.: Just let the cane hit it: how the blind and sighted see navigation differently. In Proceedings of the 16th International ACM SIGACCESS Conference on Computers \& Accessibility. ACM, pp. 217-224 (2014)

51. Tzovaras, D., Moustakas, K., Nikolakis, G., Strintzis, M.G.: Interactive mixed reality white cane simulation for the training of the blind and the visually impaired. Pers. Ubiquit. Comput. 13(1), 51-58 (2009)

52. Zhao, Y., Kupferstein, E., Tal, D., Azenkot, S.: It looks beautiful but scary: how low vision people navigate stairs and other surface level changes. In: Proceedings of the 20th International ACM SIGACCESS Conference on Computers and Accessibility. ACM, pp. 307-320 (2018)

53. Nicolau, H., Jorge, J., Guerreiro, T.: Blobby: how to guide a blind person. In: CHI'09 Extended Abstracts on Human Factors in Computing Systems. ACM, pp. 3601-3606 (2009)

54. Sandnes, F.E.: What do low-vision users really want from smart glasses? Faces, text and perhaps no glasses at all. In: International Conference on Computers Helping People with Special Needs. Springer, Cham, pp. 187-194 (2016)

55. Roentgen, U.R., Gelderblom, G.J., de Witte, L.P.: The development of an indoor mobility course for the evaluation of electronic mobility aids for persons who are visually impaired. Assist. Technol. 24(3), 143-154 (2012)

56. JASP Team: JASP (Version 0.9) [Computer software] (2018)

57. Wobbrock, J.O., Findlater, L., Gergle, D., Higgins, J.J.: The aligned rank transform for nonparametric factorial analyses using only anova procedures. In: Proceedings of the SIGCHI Conference on Human Factors in Computing Systems. ACM, pp. 143-146 (2011)

58. Alma, M.A., Van der Mei, S.F., Melis-Dankers, B.J., Van Tilburg, T.G., Groothoff, J.W., Suurmeijer, T.P.: Participation of the elderly after vision loss. Disabil. Rehabil. 33(1), 63-72 (2011)

59. Schakel, W., Bode, C., Van Der Aa, H.P., Hulshof, C.T., Bosmans, J.E., Van Rens, G.H., Van Nispen, R.M.: Exploring the patient perspective of fatigue in adults with visual impairment: a qualitative study. BMJ Open 7(8), e015023 (2017)

Publisher's Note Springer Nature remains neutral with regard to jurisdictional claims in published maps and institutional affiliations. 